\title{
Finite element analysis of the lower extrtemity - hinge knee behavior under dynamic load
}

\author{
L. Zach, S. Konvickova, P. Ruzicka \\ Department of Mechanics, Biomechanics and Mechatronics \\ CTU in Prague - Faculty of Mechanical Engineering \\ Prague, Czech Republic \\ Lukas.zach@fs.cvut.cz
}

Received: July 3, 2019. Revised: August 5, 2021. Accepted: September 12, 2021. Published: November 27, 2021.

\begin{abstract}
A key goal of joint endoprosthesis is to become a full-featured functional and anatomical replacement. The joint damage may occur for several reasons - primarily a disease of different nature and magnitude, resulting in gradual and irreversible changes and in an extreme solution in the implantation of artificial joints. However, there should be also mentioned accidents leading to joint destruction, which are often "trigger mechanism" of the disease. This work therefore presents a dynamic computational finite element analysis of a hinge-type knee replacement, which aim to streamline and accelerate the development of knee endoprosthesis. It tackles a question of the overall strength of the implant and detects sites of elevated concentrations of stresses that may be potential sources of implant damages. It also studies the behavior of the endoprosthesis under dynamic loads with emphasis on the study of the shape and size of the contact surfaces, which are closely related to the size of the contact pressure and material wear. Aside the hinged knee replacement, the computational model consisted of femur, fibula, tibia, patella and 25 most important muscles of the lower limb. Due to realistic definition of the boundary conditions, this model is suitable for investigation of invivo knee joint replacement behavior.
\end{abstract}

Keywords - Knee, knee replacement, finite element method, lower limb

\section{INTRODUCTION}

Finite element method (FEM) is a common and an effective tool used in mechanics for a development or a verification of various components or mechanisms. In biomechanics, using FEM means to undergo many compromises and simplifications. All these simplifications have to be reasonable and must take into account as many tissue characteristics as possible.

Considering this fact, there are two groups of FEA of lower extremity models. The first ones are used to simulate a behavior of a healthy knee joint in-vivo [1, 2, 3, 4] and the second group which deals with a knee joint after a total knee endoprosthesis (TKE) implantation [5,6].

The aim of this paper is to present the complex model of the lower limb, consisting of all bones of the knee and 25 main muscles of the lower limb and 8 ligament units of the knee. This complex model of lower limb model simulates dynamic behavior of the modular oncology hinged knee endoprosthesis and predicts contact pressure and stress distribution for the TKE.

\section{MATERIALS AND METHODS}

\section{A. Geometric model}

For the presented nonlinear dynamic analysis solved in Abaqus CAE, a universal size of the hinged knee endoprosthesis by ProSpon [7] was chosen.

The modular hinged knee ProSpon is made up of several components to cover individual operation demands. For the presented model, all its main components were modeled, i.e. femoral component, femoral stabilizing rod, tibial compo-nent, tibial stabilizing rod, meniscal component (tibial plat-eau), hinge post, hinge pin, hinge lock and two hinge bush-ings (see Fig. 1). Position of the TKE on the corresponding bones respected the formerly designed mechanical axis and producer's recommendations to a surgeon concerning an endoprosthesis implantation.

A bone anatomy was reconstructed based on the male cadaver CT scans of the Visible Human Project [8] provid-ed by the National Library of Medicine. A pelvic bone, necessary for muscles origins definition, was adopted from a model library of the BEL Repository, managed by the Istituti Ortopedici Rizzoli, Bologna, Italy [9].
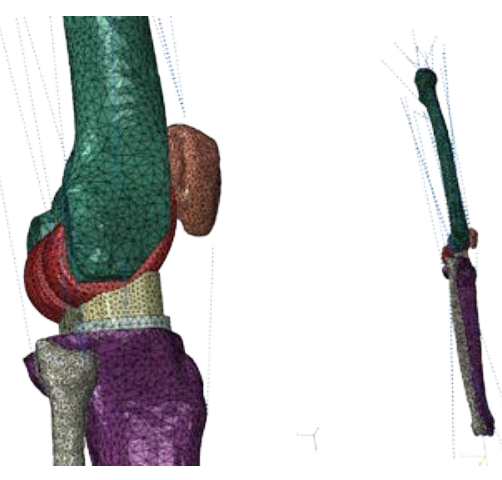

Fig. 2. Lower limb geometric model 


\section{B. Material properties and mesh generation}

For material definitions, only isotropic homogenous material models were used (see Table 1).

All materials excluding UHMWPE were supposed to behave according to Hook's low; the tibial plateau formed from UHMWPE has been defined as an elasto-plastic material (see Fig. 2). All muscles were represented by lines of actions with no material properties definitions. The patellar ligament was modeled as a spring with stiffness of $1000 \mathrm{~N} / \mathrm{mm}$.

A mash of elements was created semiautomatically using mixture of hexahedral, tetrahedral and wedge elements. The assembly consisted totally of 338003 elements.

\section{Boundary conditions}

The only contact defined in the analyses was the one between the corresponding pairs of the hinge. All other contacts were considered as tie contacts which agreed with the ideal fixation of the TKE to the bone tissue.

Magnitudes of muscle forces were adopted from Vilímek [12] who calculated by a static optimalisation muscle forces for a group of 31 musculotendon actuators. Following 25 muscles took part of the presented FEA: two parts of gluteus medius (GLMED), two parts of gluteus minimus (GLMIN), semimembranosus (SM), semitendinosus (ST), biceps femoris long head (BFL), biceps femoris short head (BFS), sartorius (SR), adductor longus (ADL), adductor breve (ADB), tensor fascia lata (TFL), pectineus (PCT), gracilis (GRC), gluteus maximus (GLMAX), ilio-psoas (ILPS), rectus femoris (RF), vastus medalis (VM), vastus intermedius (VI), vastus lateralis (VL), medial gastrocnemius (MG), lateral gastrocnemius (LG), soleus (SOL), tibialis anterior (TA) and tibialis posterior (TP).

The resulting ground reaction force was also adopted from Vilimek [12].

TABLE I. MATERIAL PROPERTIES

\begin{tabular}{lll}
\hline \multicolumn{1}{c}{ Entity (Material) } & \multicolumn{1}{c}{$\begin{array}{c}\text { Young's modulus } \\
\text { [MPa] }\end{array}$} & $\begin{array}{c}\text { Poisson's } \\
\text { ratio [-] }\end{array}$ \\
\hline Bones & 14000 & 0.36 \\
Femoral component (TiAl6V4) & 113800 & 0,34 \\
Femoral stabilizing rod (TiAl6V4) & 113800 & 0,34 \\
Tibial component (TiAl6V4) & 113800 & 0,34 \\
Tibial stabilizing rod (TiAl6V4) & 113800 & 0,34 \\
Meniscal component (UHMWPE) & 820 & 0.44 \\
Hinge post (TiAl6V4) & 113800 & 0,34 \\
Hinge pin (TiAl6V4) & 113800 & 0,34 \\
Hinge lock (TiAl6V4) & 113800 & 0,34 \\
Hinge bushings (PEEK) & 3650 & 0.44 \\
\hline
\end{tabular}

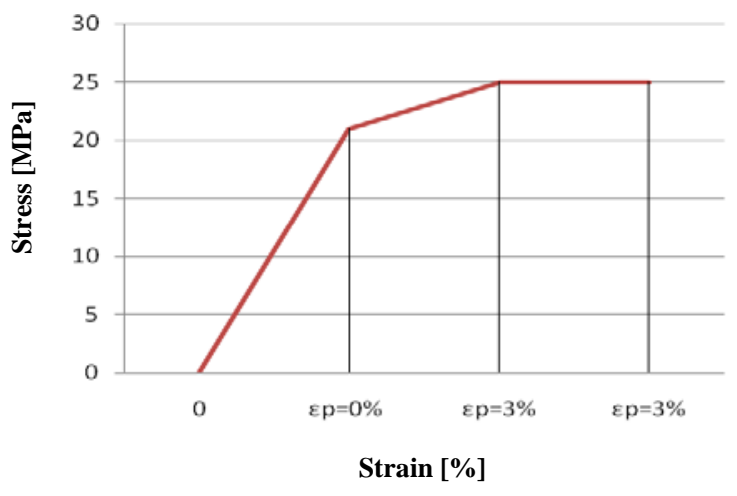

Fig. 3. UHMWPE elasto-plastic material model

All shifts and rotations but flexion were constrained in case of femoral head.

For the tibia, all rotations in an ankle were allowed as well as the proximal-distal shift.

The patella was allowed to move only in anterior-posterior axis direction which simulated a simplified articular capsule.

The dynamic FEA was run for the flexion of a hip joint up to $69.4^{\circ}$.

\section{RESULTS AND DISCUSSION}

The main goal of the FEA was to investigate an in-vivo hinge knee behavior by dynamic load. The interface boneendoprosthesis has not been studied in this analysis. The results pointed out on the most loaded parts and locations of the endoprosthesis. As supposed, the most critical component of the hinge knee is the PEEK bushing.

Fig. 3 illustrates the deformed assembly at $18.3^{\circ}\left(9.46^{\circ}\right)$, $69.4^{\circ}\left(28.3^{\circ}\right)$ and $93.1^{\circ}\left(31.3^{\circ}\right)$ of the hip joint flexion (ankle joint flexion respectively).

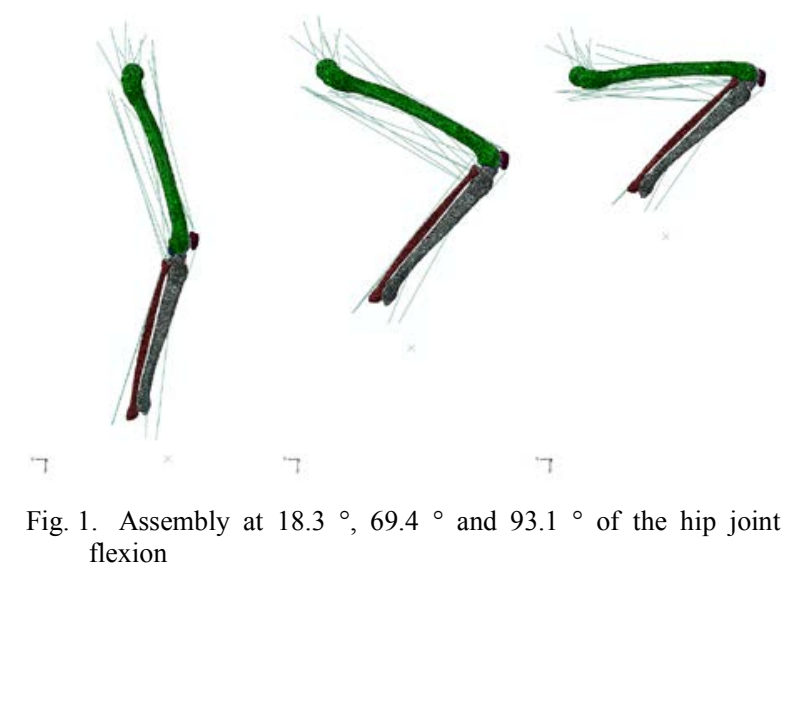


Comparison of ankle flexion - Experiment and FEA

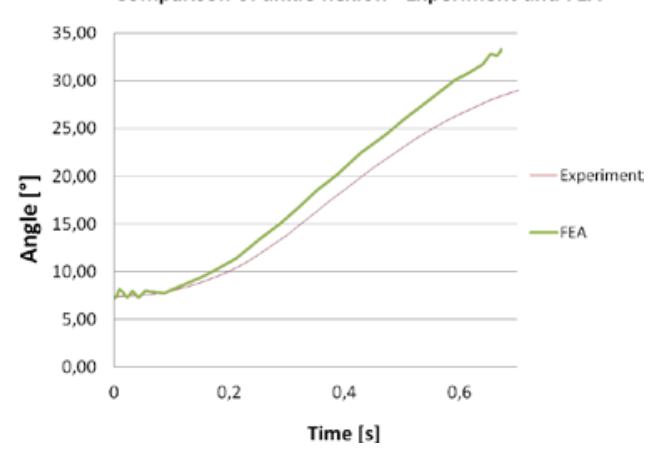

Fig. 4. Comparison of FEA and experiment [12]

To evaluate the quality of the FE simulation, we also focused on the comparison of the angle of flexion in the ankle joint calculated by FEA and experimentally measured [12]. The maximum deviation of the angle of $14 \%$ was assessed as acceptable (Fig. 4).

\section{ACKNOWLEDGMENT}

This research is supported by a grant of Technology Agency of the Czech Republic TACR01010185 and a grant of Ministry of Industry of the Czech Republic FR-TI3/221.

\section{REFERENCES}

[1] J.M.T. Penrose, "Development of an accurate three dimensional finite element knee model," in Comp. Meth. in Biomech. and Biomed. Eng. vol.5, 2002, pp. 291-300.

[2] T.L.H. Donahue, et al, "A finite element model of the Human knee joint for the study of tibio-femoral contact," in J. Biomech. Eng. vol.124, 2002, pp. 279-280.

[3] J.A. Heegaard, "A computer model to simulate patellar biomechanics following total kneee replacemnet: the effects of femoral component alignment," in Clinical Biomech. vol.16, 2001, pp. 415-423.

[4] P. Beillas, et al, "A new method to investigate in vivo knee behavior using a finite element model of the lower limb," in J Biomech. vol.37, 2004, pp. 1019-1030

[5] A.C. Godest, "Simulation of a knee joint replacement during a gait cycle using explicit finite element analysis," in J. Biomech. vol.35, 2002, pp. 267-275.

[6] J.P. Halloran, "Explicit finite element modeling of total knee replacement mechanics," in. J. Biomech. vol.38, 2004, pp. 323-331

[7] ProSpon,s.r.o. at http://www.prospon.cz

[8] National Library of Medcine, Visible Human Project at http://www.nlm.nih.gov/research/visible/visible_human.htm

[9] Viceconti, Visible Human Male - Bone surfaces, From The BEL Repository at http://www.tecno.ior.it/VRLAB/

[10] S.C. White, et al, "A Three Dimensional Musculoskeletal Model for Gait Analysis. Anatomical Variability Estimates," in J. Biomech. vol.22, 1989, pp. 885-893.

[11] R.A. Brand, et al., "A model of lower extremity muscular anatomy," in J. Biomech. Eng. vol.104, 1982, pp. 304-310.

[12] M. Vilimek, "The challenges of musculotendon forces estimation in multiple muscle systems", $\mathrm{PhD}$ Thesis. Prague, Czech Technical University in Prague - Fac. of Mechanical Engineering, 2005.

\section{Creative Commons Attribution License 4.0 (Attribution 4.0 International, CC BY 4.0)}

This article is published under the terms of the Creative Commons Attribution License 4.0 https://creativecommons.org/licenses/by/4.0/deed.en US 